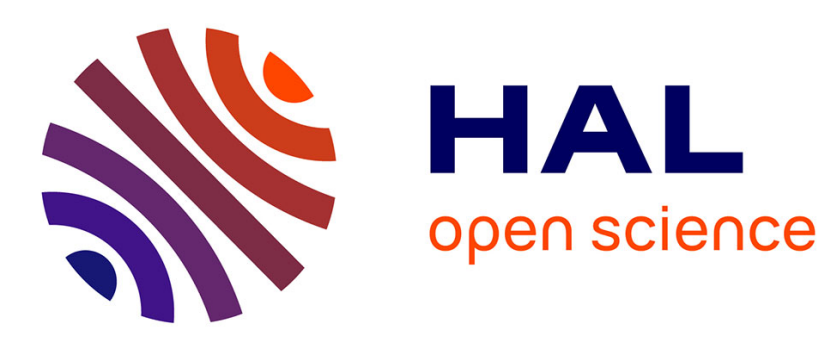

\title{
Atomic nonaffinity as a predictor of plasticity in amorphous solids
}

Bin Xu, Michael Falk, Sylvain Patinet, Pengfei Guan

\section{To cite this version:}

Bin Xu, Michael Falk, Sylvain Patinet, Pengfei Guan. Atomic nonaffinity as a predictor of plasticity in amorphous solids. Physical Review Materials, 2021, 5 (2), 10.1103/PhysRevMaterials.5.025603 . hal-03155145

\section{HAL Id: hal-03155145 \\ https://hal.science/hal-03155145}

Submitted on 1 Mar 2021

HAL is a multi-disciplinary open access archive for the deposit and dissemination of scientific research documents, whether they are published or not. The documents may come from teaching and research institutions in France or abroad, or from public or private research centers.
L'archive ouverte pluridisciplinaire HAL, est destinée au dépôt et à la diffusion de documents scientifiques de niveau recherche, publiés ou non, émanant des établissements d'enseignement et de recherche français ou étrangers, des laboratoires publics ou privés. 


\title{
Atomic nonaffinity as a predictor of plasticity in amorphous solids
}

\author{
Bin Xu $\odot,{ }^{1,2}$ Michael L. Falk, ${ }^{3, *}$ Sylvain Patinet, ${ }^{4}$ and Pengfei Guan ${ }^{1, \dagger}$ \\ ${ }^{1}$ Beijing Computational Science Research Center, Beijing 100193, China \\ ${ }^{2}$ Department of Materials Science and Engineering, Johns Hopkins University, Baltimore, Maryland 21218, USA \\ ${ }^{3}$ Departments of Materials Science and Engineering, Mechanical Engineering, and Physics and Astronomy, Johns Hopkins University, \\ Baltimore, Maryland 21218, USA \\ ${ }^{4}$ PMMH, CNRS UMR 7636, ESPCI Paris, PSL University, Sorbonne Université, Université de Paris, F-75005 Paris, France
}

(Received 16 April 2019; accepted 15 February 2021; published 26 February 2021)

\begin{abstract}
Structural heterogeneity of amorphous solids presents difficult challenges that stymie the prediction of plastic events, which are intimately connected to their mechanical behavior. Based on a perturbation analysis of the potential energy landscape, we derive the atomic nonaffinity as an indicator with intrinsic orientation, which quantifies the contribution of an individual atom to the total nonaffine modulus of the system. We find that the atomic nonaffinity can efficiently characterize the locations of the shear transformation zones, with a predicative capacity comparable to the best indicators. More importantly, the atomic nonaffinity, combining the sign of the third-order derivative of energy with respect to coordinates, reveals an intrinsic softest shear orientation. By analyzing the angle between this orientation and the shear loading direction, it is possible to predict the protocol-dependent response of one shear transformation zone. Employing this method, the distribution of orientations of shear transformation zones in model two-dimensional amorphous solids can be measured. The resulting plastic events can possibly be understood from a simple model of independent plastic events occurring at variously oriented shear transformation zones. These results shed light on the characterization and prediction of the mechanical response of amorphous solids.
\end{abstract}

DOI: 10.1103/PhysRevMaterials.5.025603

Understanding how the heterogeneity of amorphous structures correlates with mechanical response remains a significant challenge. Various indicators have been proposed to quantitatively predict where the material is susceptible to plastic transformation. Some of these only consider the structural geometry, such as free volume [1,2], fivefold symmetry $[3,4]$, local deviation from sterically favored structures [5], etc. Others of these take the interaction between particles into consideration, like low-frequency normal modes [6-9], potential energy [10], local elastic modulus [11], flexibility volume [12], mean-square vibrational amplitude (MSVA) [13], local thermal energy [14,15], local yield stress (LYS) [16,17], and saddle-point sampling [18]. Recently, machine learning has also proven to be a promising statistical tool to build a relation between structure and plastic rearrangements [19-22].

Nevertheless, most of these indicators are inherently scalar quantities, while the deformation mechanism must have an oriented shearlike character [23]. This is clearly borne out by the fact that the orientational nature of shear transformation zones (STZs), the defects purported to be associated with plastic rearrangement, can be measured through their high sensitivity to the deformation protocol. As verified in simulations, under different loading orientations, the same glass may exhibit contrasting mechanical responses during which entirely different STZs are activated [14,16-18,24].

\footnotetext{
*mfalk@jhu.edu

†pguan@csrc.ac.cn
}

Obtaining the mechanical response along different orientations of one STZ may be accomplished in a number of ways: by measuring the LYS $[16,17,25]$, by calculating the linear response of local thermal energy with respect to strain (LRLTE) [15], or by sampling low-energy events [18]. All of these methods require computationally expensive calculations. LYS requires prior calculations to determine the appropriate probing length scale and direct computation of response along many orientations [17]. LRLTE must be recalculated under the specified mechanical load to compare different orientations [15]. Sampling low-energy events only captures the subset of events that are inherently viscoplastic, and requires the harvesting of large numbers of events so as to find the few lowest-energy events.

In this paper, based on a perturbation analysis of the energy landscape, we derive a parameter-free and low-cost indicator, termed the atomic nonaffinity. Since this indicator is derived from a perturbation method, the atomic nonaffinity can precisely predict the mechanical behavior near the reference state and becomes less effective as the system is deformed. We show that atomic shear nonaffinity, i.e., the shear part of the atomic nonaffinity, can efficiently predict the locations of plastic rearrangements during shear deformation of twodimensional amorphous solids with an accuracy comparable to the best known indicators. The relevant orientational information of STZs is naturally reflected in this parameter, and analysis of the atomic shear nonaffinity indicates that the softest shear orientation of the triggered STZs aligns with the orientation of the applied shear protocol. Moreover, the 
(a)

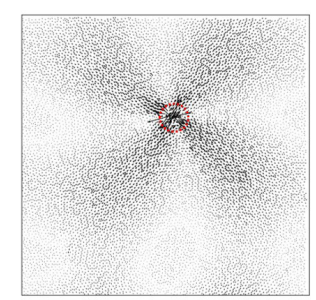

(b)

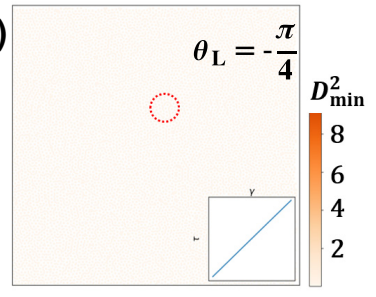

(c)

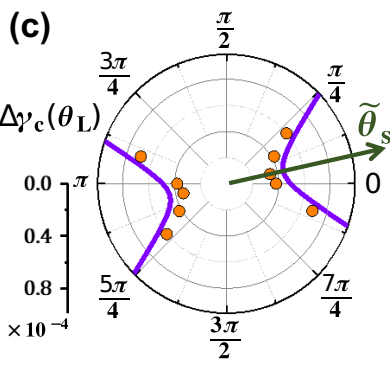

(e)

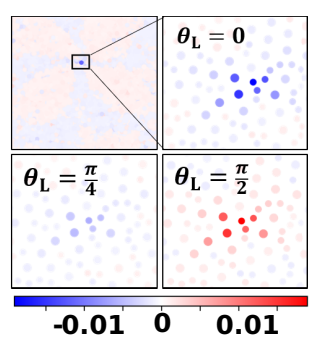

(d)

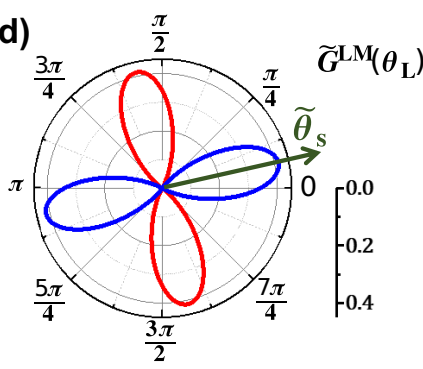

(f)

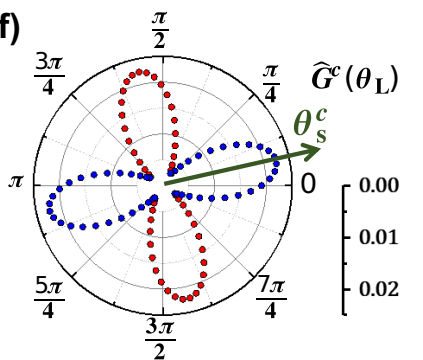

FIG. 1. Analysis of configuration that was sheared to be close to the triggering of a plastic event. (a) The spatial distribution of the normal mode with lowest eigenvalue. (b) The $D_{\min }^{2}$ field [26] after shear with strain of $\Delta \gamma=6 \times 10^{-5}$ in the orientation of $\theta_{\mathrm{L}}=-\pi / 4$. The inset shows the stress-strain curve. (c) The predicted triggering strain (line) and triggering strain from simulation (circles) as a function of shear angle $\theta_{\mathrm{L}}$. (d) The magnitude of nonaffine modulus contribution from the lowest mode at different $\theta_{\mathrm{L}}$. The blue line represents the range of $\theta_{\mathrm{L}}$, where the plastic event can be triggered, while the red line represents the range of $\theta_{\mathrm{L}}$, where the plastic event cannot be triggered. (e) The spatial distribution of $\hat{G}\left(\theta_{\mathrm{L}}\right) \operatorname{sign}\left(\Delta \gamma_{c}\left(\theta_{\mathrm{L}}\right)\right)$ for different orientations. (f) The atomic shear nonaffinity in different orientations for the atom that has the maximum magnitude of atomic shear nonaffinity in (e).

distribution of orientations of activated STZs is calculated, and we show that this distribution can be understood through a simple model that assumes independent STZs with isotropically distributed soft orientations.

To motivate the relevance of the atomic nonaffinity we first consider a special state in which a two-dimensional amorphous system is deformed to be close to the triggering strain of a plastic event via a protocol of athermal quasistatic shear. The two-dimensional glassy system, comprised of $10^{4}$ particles, was prepared via the same gradual quench and the same smoothed Lennard-Jones potential described in Refs. [16,17]. The spatial distribution of the normal mode with the lowest eigenvalue, referred to here as the lowest mode (LM), is shown in Fig. 1(a). A plastic event will be triggered in the region (see Fig. S1 in the Supplemental Material (SM) [27]) where the LM is localized if the system is further sheared in this direction, denoted as the reference direction, $\theta_{\mathrm{L}}=0$. However, if the system is further sheared with similar small

strains or even larger ones in other directions, such as $\theta_{\mathrm{L}}=$ $-\frac{\pi}{4}$ or $\frac{\pi}{4}$, the triggering of the same plastic event is not observed [see Fig. 1(b) and Fig. S1 in the SM]. Obviously, this protocol-dependent mechanical behavior of amorphous systems cannot be clearly understood solely with scalar indicators. Here, we introduce the second and the third derivatives of the energy with respect to the vibrational coordinate $\left(q^{*}\right)$ of the LM, denoted as $\lambda^{*}$ and $\eta^{*}$, respectively, and the first derivative of stress of the system with respect to $q^{*}$ (denoted as $\frac{\partial \sigma_{x x}}{\partial q^{*}}, \frac{\partial \sigma_{y y}}{\partial q^{*}}$, and $\frac{\partial \tau_{x y}}{\partial q^{*}}$, respectively). The triggering strain for different shear orientations can be derived as (see the SM [27] for details of derivation)

$$
\Delta \gamma_{c}\left(\theta_{\mathrm{L}}\right)=\frac{\lambda^{* 2}}{2 \eta^{*} V \frac{\partial \tau\left(\theta_{\mathrm{L}}\right)}{\partial q^{*}}},
$$

where $V$ is the volume of the system and $\frac{\partial \tau\left(\theta_{L}\right)}{\partial q^{*}}$ is the first derivative of the shear stress with respect to $q^{*}$ at $\theta_{\mathrm{L}}$, which is equal to $-\frac{1}{2}\left(\frac{\partial \sigma_{x x}}{\partial q^{*}}-\frac{\partial \sigma_{y y}}{\partial q^{*}}\right) \sin 2 \theta_{\mathrm{L}}+\frac{\partial \tau_{x y}}{\partial q^{*}} \cos 2 \theta_{\mathrm{L}}$. A similar form was also obtained from prior analyses of the plastic mode [28]. Moreover, a softest shear orientation of the LM, $\tilde{\theta}_{s}$, associated with the smallest positive triggering strain can be defined as

$$
\begin{gathered}
\tan 2 \tilde{\theta}_{s}=-\left(\frac{\frac{\partial \sigma_{x x}}{\partial q^{*}}-\frac{\partial \sigma_{y y}}{\partial q^{*}}}{\frac{2 \partial \tau_{x y}}{\partial q^{*}}}\right), \text { with } \\
\frac{\partial \tau\left(\theta_{s}\right)}{\partial q^{*}} \eta^{*}>0 \text { and } \theta_{s} \in\left(-\frac{\pi}{2}, \frac{\pi}{2}\right] .
\end{gathered}
$$

Here we take the symmetry of shear into consideration and note that shear with orientation of $\theta_{s}$ is equal to shear with orientation of $\theta_{s}+\pi$. To verify the validity of the predictions of Eqs. (1) and (2), further simulations were performed to directly measure $\Delta \gamma_{c}\left(\theta_{\mathrm{L}}\right)$ and $\tilde{\theta}_{s}$. As shown in Fig. 1(c), the predictions agree well with the simulation results, which suggests that the analysis of LM is successful for calculating the orientation-dependent mechanical response of the system close to the instability. When the system is far from the instability, we suppose that all the modes, especially the ones with small eigenvalues, should be taken into consideration.

To develop an indicator that takes all modes into consideration while maintaining the orientational information of each mode, we investigate how different modes contribute to the system modulus. Following Maloney [6,29], the elastic constants of amorphous solids can be derived from the second derivative of the total potential energy with respect to strain in athermal quasistatic deformation. These can be rewritten in the coordinates of the eigenbasis as

$$
C_{i j k l}=\frac{1}{V}\left(\frac{\partial^{2} U}{\partial \epsilon_{i j} \partial \epsilon_{k l}}+\sum_{m} \frac{\partial^{2} U}{\partial q_{m} \partial \epsilon_{i j}} \frac{d q_{m}}{d \epsilon_{k l}}\right),
$$

where $U$ is the potential energy, and $q_{m}$ is the $m$ th coordinate of the eigenbasis of the Hessian matrix $\left(\frac{\partial^{2} U}{\partial r_{0} \partial r_{0 j}}\right)$. The first term (Born term) of Eq. (3) accounts for affine displacement and is insensitive to the structural stability [30]. The second term, containing the contribution from nonaffine relaxation in each normal mode, termed the nonaffine modulus $(\tilde{C})$ here, is sensitive to the structural stability. By expressing the stress 
as $\sigma_{i j}=\frac{1}{V} \frac{\partial U}{\partial \epsilon_{i j}}$ and the nonaffine "velocity" in quasistatic deformation as $\frac{d q_{m}}{d \epsilon_{k l}}=-\frac{1}{\lambda_{m}} \frac{\partial \sigma_{k l}}{\partial q_{m}}$ [6], where $\lambda_{m}$ is the eigenvalue of the $m$ th normal mode, the nonaffine part, $\tilde{C}_{i j k l}$, can be rewritten as

$$
\tilde{C}_{i j k l}=\sum_{m} \tilde{C}_{i j k l, m}=\sum_{m}-\frac{V}{\lambda_{m}} \frac{\partial \sigma_{i j}}{\partial q_{m}} \frac{\partial \sigma_{k l}}{\partial q_{m}},
$$

where $\tilde{C}_{i j k l, m}$ is the contribution from the $m$ th normal mode, which is always negative. In shear protocols, the shear modulus is the most important elastic constant. Thus, we focus on the nonaffine shear modulus $(\tilde{G})$ and the contribution from each mode $\left(\tilde{G}_{m}\right)$. The $\tilde{G}_{m}$ can be calculated by

$$
\tilde{G}_{m}\left(\theta_{\mathrm{L}}\right)=-\frac{V}{\lambda_{m}}\left(\frac{\partial \tau\left(\theta_{\mathrm{L}}\right)}{\partial q_{m}}\right)^{2},
$$

which depends on the orientation $\theta_{\mathrm{L}}$. The nonaffine shear modulus contribution of the dominant LM, $\tilde{G}^{\mathrm{LM}}$, for the state described in Figs. 1(a)-1(c) is shown in Fig. 1(d). The blue line represents the orientational range, where $\Delta \gamma_{c}>0$, i.e., $\frac{\partial \tau\left(\theta_{\mathrm{L}}\right)}{\partial q^{*}} \eta^{*}>0$ following Eq. (1), and the event can be triggered. The red line represents the orientational range, where the event cannot be triggered. Moreover, a softest shear orientation can also be defined by the largest value of $\tilde{G}^{\mathrm{LM}}$ in the blue range which is and should be consistent with the $\tilde{\theta}_{s}$ derived from Eq. (2).

So far our results have been discussed with respect to eigenbasis. To develop an indicator expressed in terms of atomic quantities, we borrow an idea from the literature regarding the participation fraction [7,9]. By expressing the normalized eigenvector in the atomic coordinates as $\boldsymbol{\Psi}_{m}=$ $\sum_{n, \alpha} c_{m n \alpha} \boldsymbol{e}_{n \alpha}$, where $\boldsymbol{e}_{n \alpha}$ is a unit vector corresponding to the displacement of the $n$th atom in the $\alpha(=x$ or $y)$ direction, and $c_{m n \alpha}$ is the projection of the $m$ th eigenvector onto $\boldsymbol{e}_{n \alpha}$, the $\tilde{C}_{i j k l}$ can be rewritten as

$$
\tilde{C}_{i j k l}=\sum_{n} \hat{C}_{i j k l, n}=\sum_{n} \sum_{m, \alpha}-\frac{V}{\lambda_{m}} \frac{\partial \sigma_{i j}}{\partial q_{m}} \frac{\partial \sigma_{k l}}{\partial q_{m}} c_{m n \alpha}^{2} .
$$

Here $\hat{C}_{i j k l, n}$ is the atomic nonaffinity of the $n$th atom. As most local plastic rearrangements are shearlike [26,31], the atomic shear nonaffinity (ASN) is the most important component when investigating the STZs and can be written as

$$
\hat{G}_{n}\left(\theta_{\mathrm{L}}\right)=\sum_{m, \alpha}-\frac{V}{\lambda_{m}}\left(\frac{\partial \tau\left(\theta_{\mathrm{L}}\right)}{\partial q_{m}}\right)^{2} c_{m n \alpha}^{2} .
$$

Obviously, the value of $\hat{G}_{n}$ depends on the orientation $\theta_{\mathrm{L}}$. As a result, the spatial distribution of $\hat{G}_{n}$ in the previous case shown in Fig. 1(e) exhibits a clear orientation-dependent behavior in the region where the plastic event is located. More negative values of $\hat{G}_{n}$ mean that the corresponding atom is easier to trigger in the orientation $\theta_{\mathrm{L}}$. The $\hat{G}_{n}$ distribution calculated at different orientations indicates that $\theta_{\mathrm{L}}=0$ is the easiest shear direction for the plastic event when compared with $\theta_{\mathrm{L}}=\frac{\pi}{4}$ and $\frac{\pi}{2}$, which is consistent with the direct loading test in Fig. 1(b) and Fig. S1 in the SM [27]. Moreover, the atom located in the core region has the maximum magnitude of atomic shear nonaffinity, denoted as $\hat{G}^{c}\left(\theta_{\mathrm{L}}\right)$. Figure $1(\mathrm{f})$ shows the $\theta_{\mathrm{L}}$-dependent $\hat{G}^{c}$, which has a similar shape as the $\tilde{G}^{\mathrm{LM}}$. This is attributed to the fact that the LM with the smallest eigenvalue dominates the variation of $\hat{G}^{c}$, which can be inferred from Eq. (7). Thus, we can define the softest shear orientation for the $n$th atom as the softest shear orientation of the mode that dominates the variation of $\hat{G}_{n}$. The softest shear orientation of the $n$th atom is defined as

$$
\theta_{n, s}=\tilde{\theta}_{i, s}, \quad i=\operatorname{argmax}_{m} \sum_{\alpha}\left|\tilde{G}_{m}\left(\tilde{\theta}_{m, s}\right)\right| c_{m n \alpha}^{2},
$$

and the calculated softest shear orientation of the core atom $\left(\theta_{s}^{c}\right)$ is shown in Fig. 1(f). The consistency of the proposed softest shear orientations for one STZ from the three parameters, i.e., the directly calculated triggering strain [Fig. 1(c)], the nonaffine modulus of the lowest mode [Fig. 1(d)], and the atomic shear nonaffinity [Fig. 1(f)], implies that the $\theta_{s}$ defined from atomic shear nonaffinity is effective for characterizing the orientations of STZs.

Now that we have seen the predictive capacity of atomic shear nonaffinity regarding the protocol-dependent mechanical response of a plastic event close to instability, we can ask, "What if the system is not close to instability?" and "How predictive is this indicator?" Predicting plastic events in an amorphous system by analyzing the local indicators of initial structure has been extensively studied in the literature $[7-9,14,16,18,24,28,32,33]$. To compare the reliability of local indicators for predicting plastic events, 100 twodimensional samples prepared with the same thermal history as the previous sample were employed for local properties calculations. The athermal quasistatic shear deformation with a strain step of $\Delta \gamma_{x y}=10^{-5}$ was then applied to each sample, and each stress drop in the stress-strain curve was associated with the resulting atomic rearrangements corresponding to one plastic event. The nonaffine rate [6] was calculated for the configurations just before the stress drops, and the atom with maximum nonaffine rate was identified as the core atom, whose index is denoted as $\operatorname{ID}_{N}$ for the $N$ th plastic event. To compare the success of different indicators, we transform those indicators to a rank correlation (RC) value following the analysis performed by Patinet et al. [16] as

$$
\mathrm{RC}_{\Psi}(n)=1-2 \mathrm{CDF}_{\Psi}(n),
$$

where $\Psi$ is one of the indicators, $\mathrm{CDF}_{\Psi}$ is the cumulative distribution function for the $\Psi$ of all atoms, and $\mathrm{CDF}_{\Psi}(n)$ is the function value in the range of $[0,1]$ based on the value of $\Psi$ for the $n$th particle. The spatial distribution field of the calculated $\mathrm{RC}_{\hat{G}}$ with $\theta_{\mathrm{L}}=0$ is shown in Fig. 2(a). The first ten plastic events in shear protocols with $\theta_{\mathrm{L}}=0$ are almost all located at high- $\mathrm{RC}_{\hat{G}}$ regions, which implies the highly predictive power of $\hat{G}$. To quantitatively compare the predictive power regarding plastic events for different local indicators, the relationship between the locations of plastic events and the corresponding values of local indicators is described by the average of $\mathrm{RC}_{\Psi}\left(\mathrm{ID}_{N}\right)$ over 100 samples. The average $\mathrm{RC}_{\Psi}\left(\mathrm{ID}_{N}\right)$ of investigated local indicators, such as the participation fraction (PF) [7-9] in the lowest $1 \%$ of normal modes, the nonaffine rate (NR) [6], the MSVA [13], the LYS [16], and the ASN, are shown in Fig. 2(b). The LYS presents the highest predictive power in the early stage, since nonlinear response to shear is considered. The MSVA and ASN show comparable predictive power, and the other indicators have 

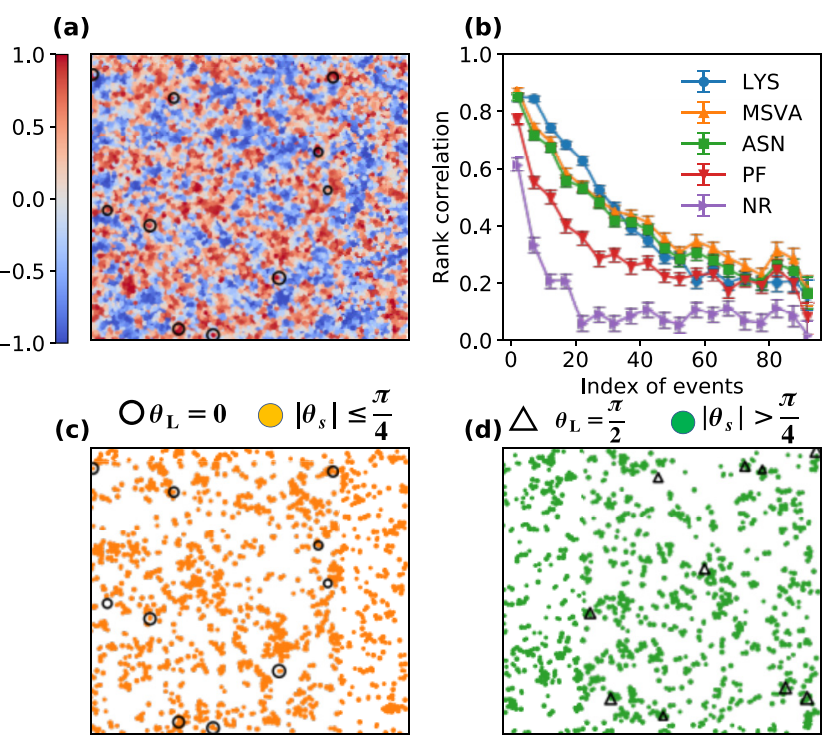

FIG. 2. Predicting plastic events by analyzing initial configurations. (a) The correlation field of atomic shear nonaffinity $\hat{G}\left(\theta_{\mathrm{L}}=\right.$ $0)$ and the locations of the first ten plastic events (black circles) triggered in shear protocols with $\theta_{\mathrm{L}}=0$. (b) Correlation between the indicators including local yield stress (LYS), mean-square vibrational amplitude (MSVA), atomic shear nonaffinity (ASN), participation fraction (PF), and nonaffine rate (NR) with the locations of plastic events as a function of the index of the events. Averages are taken over windows of five events. The error bar at each window represents the standard deviation of the mean. (c) Orange circles represent the atoms with $\mathrm{RC}_{\hat{G}}>0$ and $\left|\theta_{s}\right|<\frac{\pi}{4}$. Black circles mark the locations of the first ten plastic events with $\theta_{\mathrm{L}}=0$. (d) Green circles represent the atoms with $\mathrm{RC}_{\hat{G}}>0$ and $\left|\theta_{s}\right|>\frac{\pi}{4}$. Triangles mark the locations of the first ten plastic events with $\theta_{\mathrm{L}}=\frac{\pi}{2}$.

lower predictive power than those three. It is worth noting that the predictive power of the indicators depends on the stability of configurations. In the SM [27] we show the predictive power of these indicators for configurations prepared by instantly quenching from high-temperature liquids systems in which MSVA and ASN outperform LYS. We also note that the orientational information in ASN is incomplete and it, as a modulus, has the same value for $\theta_{\mathrm{L}}=0$ and $\frac{\pi}{2}$, while local regions generally have different mechanical behavior for those two protocols.

As discussed in Fig. 1, we expect that the plastic events induced when shearing along direction $\theta_{\mathrm{L}}$ should be located at the atoms with $\left|\theta_{s}-\theta_{\mathrm{L}}\right|<\frac{\pi}{4}$, and here we test this expectation in one of the previous samples. We focused on the "soft" atoms in the sample with $\mathrm{RC}_{\hat{G}}>0$, and distinguished them by the value of $\theta_{s}$. The correlation between the atom with $\mathrm{RC}_{\hat{G}}>0$ and $\left|\theta_{s}\right|<\frac{\pi}{4}\left(\left|\theta_{s}\right|>\frac{\pi}{4}\right)$ and the first ten plastic events of $\theta_{\mathrm{L}}=0\left(\theta_{\mathrm{L}}=\frac{\pi}{2}\right)$ direction is illustrated in Fig. 2(c) [Fig. 2(d)]. The correlation in both Figs. 2(c) and 2(d) indicates that the predictive power can potentially be increased by screening for regions where the intrinsic softest orientation of $\hat{G}_{n}$ aligns with the deformation protocol. (Similar results about protocols of $\theta_{\mathrm{L}}=\frac{\pi}{4}$ and $-\frac{\pi}{4}$ are shown in the SM [27].)

However, there still exist some number of events that are not caught by the criterion $\left|\theta_{s}-\theta_{\mathrm{L}}\right|<\frac{\pi}{4}$. This can be at-
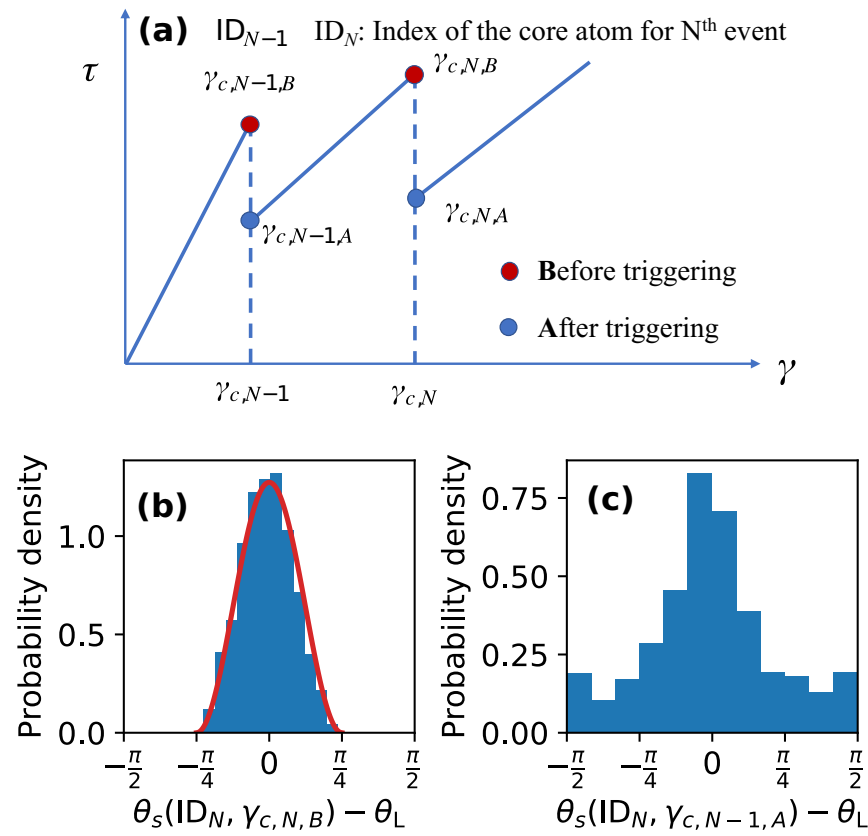

FIG. 3. Distribution of the softest shear orientations for plastic regions. (a) A schematic diagram introducing the notations used in this figure. (b) The distribution of the softest shear orientations for all the plastic events that are triggered before shear strain 0.12 in ten samples. The softest shear orientations are calculated based on the configuration just before each event. The red line follows the function $\frac{4}{\pi} \cos ^{2}(2 \Delta \theta)$. (c) Similar to (b), but the softest shear orientations for each event are calculated based on the configurations just after the previous event.

tributed to the rotation of $\theta_{s}$ during deformation, since the $\theta_{s}$ is calculated mainly based on a second-order perturbation method, and higher-order terms and nonlinear interactions between different modes can lead to the rotation of $\theta_{s}$. To obtain the statistics of the rotation of $\theta_{s}$, the softest shear orientations of core particles of all the plastic events before shear strain 0.12 with $\theta_{\mathrm{L}}=0$ in ten samples are calculated based on the configurations just before each event or just after the last event [illustrated in Fig. 3(a)]. The distribution of the calculated $\theta_{s}$ of those core particles in the configurations just before triggering are shown in Fig. 3(b) and all $\theta_{s}$ satisfy the criterion $\left|\theta_{s}-\theta_{\mathrm{L}}\right|<\frac{\pi}{4}$, which is what we expected for systems close to instability, as discussed previously. Moreover, the peak of probability density is located at $\theta_{\mathrm{L}}$, implying that the region with the intrinsically softest orientation closest to the imposed shear orientation is easiest to trigger. However, the distribution is broadened as shown in Fig. 3(c) for the calculated $\theta_{s}^{c}$ based on the configurations just after the triggering of the previous event. In this analysis only approximately $75 \%$ of the plastic events satisfy the criterion. More statistics about how orientations calculated by our perturbation method change are presented in the SM [27]. Because plastic events tend to happen at STZs closely aligned with the orientation of the shear protocols, we also show that the precision of prediction for different indicators can be improved by screening for potential STZs with the softest shear orientations in the SM [27].

The distribution of orientations of the triggered plastic event shown in Fig. 3(b) is regular. It can be understood by a 
simple model of independent plastic events with intrinsic orientations. In this model, we assume that amorphous solids are isotropic and that the shear-orientation-dependent triggering strain can be derived from Eq. (1) as

$$
\gamma_{c}\left(\theta_{\mathrm{L}}\right)=\frac{\gamma_{c}\left(\theta_{s}\right)}{\cos \left[2\left(\theta_{s}-\theta_{\mathrm{L}}\right)\right]},
$$

where $\theta_{s}$ is the softest shear orientation of a STZ. If we assume that the number density for a particular softest shear direction $\theta_{s}$ at different triggering strains $\gamma_{c}\left(\theta_{s}\right)$ (noted as $\gamma_{c, s}$ ) follows a power law $\rho\left(\gamma_{c, s}\right)=A \gamma_{c, s}^{\alpha}$ [34-37], the probability density distribution of orientations $\hat{\rho}\left(\theta_{s}-\theta_{\mathrm{L}}\right)$ [denoted as ]) will follow (see the SM [27] for details of derivation)

$$
\hat{\rho}(\Delta \theta)=k \cos ^{\alpha+1}(2 \Delta \theta) .
$$

The probability density distribution in Fig. 3(b) corresponds to $\alpha=1$, as it closely fits a distribution function $\frac{4}{\pi} \cos ^{2}(2 \Delta \theta)$ [red line in Fig. 3(b)]. These results are also supported by the probability distribution function of local yield stress of the samples, in which $\alpha \approx 1.1$, as shown in Ref. [16].

In summary, we have derived a general and parameter-free indicator, the atomic nonaffinity. It is well defined and is easy to apply in systems beyond the two-dimensional LennardJones system discussed here. The atomic nonaffinity has a clear physical meaning in that the summation of atomic nonaffinities corresponds to the total nonaffine modulus of the system. The softest shear orientation of each region is defined based on the atomic shear nonaffinity and stems from anisotropy of the shear stress derivative against the coordinate of the low-frequency mode in different orientations. When combined with the sign of the third-order derivative of energy with respect to coordinates, it reveals the intrinsic orientation of the plastic rearrangement and directly connects to the anisotropic mechanical response of local regions, which is important for understanding aspects of the mechanical behavior of amorphous solids not directly reflected or defined in other indicators. As atomic nonaffinity is developed based on the nonaffine response of atoms upon deformation, it naturally has a good correlation with the plastic events, comparable to the best indicators. Mechanical behavior must be correlated with structure, and we anticipate that this method will be important for elucidating the structural origin of the anisotropic mechanical response in specific systems.

B.X and P.F.G acknowledge financial support by the Science Challenge Project (Grant No. TZ2018004) and the National Natural Science Foundation of China (NSFC, Grant No. U1930402). M.L.F acknowledges support provided by NSF Grant Award No. 1910066/1909733. We also acknowledge the computational support from Beijing Computational Research Center. Insightful discussions with members of the "Beijing Metallic Glass Club" are highly acknowledged by B.X and P.F.G.
[1] F. Spaepen, Acta Metall. 25, 407 (1977).

[2] F. Zhu, A. Hirata, P. Liu, S. Song, Y. Tian, J. Han, T. Fujita, and M. Chen, Phys. Rev. Lett. 119, 215501 (2017).

[3] M. Wakeda, Y. Shibutani, S. Ogata, and J. Park, Intermetallics 15, 139 (2007).

[4] Y. Hu, F. Li, M. Li, H. Bai, and W. Wang, Nat. Commun. 6, 8310 (2015).

[5] H. Tong and H. Tanaka, Phys. Rev. X 8, 011041 (2018).

[6] C. Maloney and A. Lemaître, Phys. Rev. Lett. 93, 195501 (2004).

[7] A. Widmer-Cooper, H. Perry, P. Harrowell, and D. R. Reichman, Nat. Phys. 4, 711 (2008).

[8] M. L. Manning and A. J. Liu, Phys. Rev. Lett. 107, 108302 (2011).

[9] J. Ding, S. Patinet, M. L. Falk, Y. Cheng, and E. Ma, Proc. Natl. Acad. Sci. USA 111, 14052 (2014).

[10] Y. Shi, M. B. Katz, H. Li, and M. L. Falk, Phys. Rev. Lett. 98, 185505 (2007).

[11] M. Tsamados, A. Tanguy, C. Goldenberg, and J.-L. Barrat, Phys. Rev. E 80, 026112 (2009).

[12] J. Ding, Y.-Q. Cheng, H. Sheng, M. Asta, R. O. Ritchie, and E. Ma, Nat. Commun. 7, 13733 (2016).

[13] H. Tong and N. Xu, Phys. Rev. E 90, 010401(R) (2014).

[14] J. Zylberg, E. Lerner, Y. Bar-Sinai, and E. Bouchbinder, Proc. Natl. Acad. Sci. USA 114, 7289 (2017).

[15] Z. Schwartzman-Nowik, E. Lerner, and E. Bouchbinder, Phys. Rev. E 99, 060601(R) (2019).

[16] S. Patinet, D. Vandembroucq, and M. L. Falk, Phys. Rev. Lett. 117, 045501 (2016).
[17] A. Barbot, M. Lerbinger, A. Hernandez-Garcia, R. GarcíaGarcía, M. L. Falk, D. Vandembroucq, and S. Patinet, Phys. Rev. E 97, 033001 (2018).

[18] B. Xu, M. L. Falk, J. F. Li, and L. T. Kong, Phys. Rev. Lett. 120, 125503 (2018).

[19] S. S. Schoenholz, E. D. Cubuk, D. M. Sussman, E. Kaxiras, and A. J. Liu, Nat. Phys. 12, 469 (2016).

[20] Q. Wang and A. Jain, Nat. Commun. 10, 5537 (2019).

[21] V. Bapst, T. Keck, A. Grabska-Barwińska, C. Donner, E. D. Cubuk, S. S. Schoenholz, A. Obika, A. W. R. Nelson, T. Back, D. Hassabis, and P. Kohli, Nat. Phys. 16, 448 (2020).

[22] Z. Fan, J. Ding, and E. Ma, Mater. Today 40, 48 (2020).

[23] A. Nicolas and J. Rottler, Phys. Rev. E 97, 063002 (2018).

[24] O. Gendelman, P. K. Jaiswal, I. Procaccia, B. Sen Gupta, and J. Zylberg, Europhys. Lett. 109, 16002 (2015).

[25] S. Patinet, A. Barbot, M. Lerbinger, D. Vandembroucq, and A. Lemaître, Phys. Rev. Lett. 124, 205503 (2020).

[26] M. L. Falk and J. S. Langer, Phys. Rev. E 57, 7192 (1998).

[27] See Supplemental Material at http://link.aps.org/supplemental/ 10.1103/PhysRevMaterials.5.025603 for (1) The mechanical behavior of different shear orientations for one plastic event close to instability. (2) Derivation of the orientation-dependent triggering strain of the softest mode. (3) Predicative power of indicators for configurations instantly quenched from high temperature liquids. (4) Locations of plastic events with different shear protocols. (5) The statistics for the rotation of the softest shear orientation during deformation. (6) Improving the prediction of indicators via screening with the softest shear 
orientation. (7) A simple model of independent plastic events with intrinsic orientations.

[28] E. Lerner, Phys. Rev. E 93, 053004 (2016).

[29] T. Barron and M. Klein, Proc. Phys. Soc. 85, 523 (1965).

[30] Y. Q. Cheng and E. Ma, Phys. Rev. B 80, 064104 (2009).

[31] A. Argon, Acta Metall. 27, 47 (1979).

[32] E. D. Cubuk, S. S. Schoenholz, J. M. Rieser, B. D. Malone, J. Rottler, D. J. Durian, E. Kaxiras, and A. J. Liu, Phys. Rev. Lett. 114, 108001 (2015).

[33] D. Richard, M. Ozawa, S. Patinet, E. Stanifer, B. Shang, S. A. Ridout, B. Xu, G. Zhang, P. K. Morse, J.-L. Barrat, L. Berthier,
M. L. Falk, P. Guan, A. J. Liu, K. Martens, S. Sastry, D. Vandembroucq, E. Lerner, and M. L. Manning, Phys. Rev. Mater. 4, 113609 (2020).

[34] S. Karmakar, E. Lerner, and I. Procaccia, Phys. Rev. E 82, 055103(R) (2010).

[35] H. G. E. Hentschel, P. K. Jaiswal, I. Procaccia, and S. Sastry, Phys. Rev. E 92, 062302 (2015).

[36] J. Lin, E. Lerner, A. Rosso, and M. Wyart, Proc. Natl. Acad. Sci. USA 111, 14382 (2014).

[37] J. Lin, A. Saade, E. Lerner, A. Rosso, and M. Wyart, Europhys. Lett. 105, 26003 (2014). 


\section{Supplementary Materials for "Atomic nonaffinity as a predictor of plasticity in amorphous solids"}

3

Bin Xu, Michael L. Falk, Sylvain Patinet, and Pengfei Guan

\section{${ }_{4}$ I. CLOSE TO INSTABILITY OF ONE PLASTIC EVENT}

5 The two-dimensional system mentioned in the main text was sheared to be very close to 6 the triggering strain of a plastic event via a protocol of athermal quasistatic shear. A plastic 7 event will be triggered in the region (Fig. S1(a)) where the mode with lowest eigenvalue is s localized, if the system were to be further sheared in the pre-sheared direction (the reference 9 direction $\left.\theta_{\mathrm{L}}=0\right)$. However, if the system were to be further sheared in other directions, 10 such as $\theta_{\mathrm{L}}=\frac{\pi}{4}$, no plastic event would be observed in the region, as shown in Fig. S1(b), 12 even when subjected to a large magnitude of strain.

\section{${ }_{13}$ II. TRIGGERING STRAIN OF THE SOFTEST MODE}

14 In athermal quasistatic shear (AQS), the system is always mechanically stable and must 15 reside at a minimum on the energy landscape. The energy of system $U$ can be expanded 16 with a third-order Taylor approximation based on the vibrational coordinate $q^{*}$ of the softest 17 mode and shear strain as
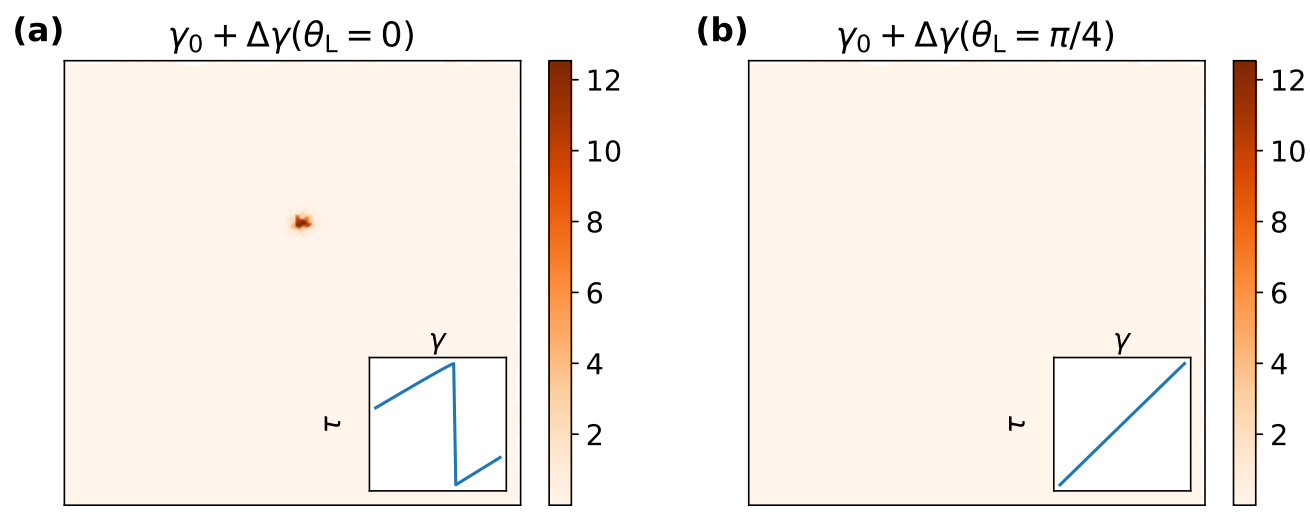

FIG. S1. The $D_{\text {min }}^{2}$ field after simple shear with $\theta_{\mathrm{L}}=0$ (a) and $\theta_{\mathrm{L}}=\pi / 4$ (b). The loading shear strain was $6 \times 10^{-5}$. A plastic event was triggered when $\theta_{\mathrm{L}}=0$. 


$$
\begin{aligned}
U \approx U_{0} & +\frac{\partial U}{\partial \gamma} \Delta \gamma+\frac{1}{2} \frac{\partial^{2} U}{\partial \gamma^{2}} \Delta \gamma^{2}+\frac{\lambda^{*}}{2} \Delta q^{* 2}+V \Delta \gamma \Delta q^{*} \frac{\partial \tau\left(\theta_{\mathrm{L}}\right)}{\partial q^{*}}+\frac{\eta^{*}}{6} \Delta q^{* 3} \\
& +\frac{1}{2} \frac{\partial^{3} U}{\partial q^{* 2} \partial \gamma} \Delta q^{* 2} \Delta \gamma+\frac{1}{2} \frac{\partial^{3} U}{\partial q^{*} \partial \gamma^{2}} \Delta q^{*} \Delta \gamma^{2}+\frac{1}{6} \frac{\partial^{3} U}{\partial \gamma^{3}} \Delta \gamma^{3}
\end{aligned}
$$

18 where $\lambda^{*}$ is the eigenvalue, $\eta^{*}$ is the third derivative of energy with respect to coordinate ${ }_{19} q^{*}, V$ is the volume of the system, $\tau\left(\theta_{\mathrm{L}}\right)$ is the shear stress in the shear orientation $\theta_{\mathrm{L}}$, and ${ }_{20} \Delta \gamma=\gamma-\gamma_{0}$ is the shear strain. We can further formulate the following assumptions:

21

22

23

${ }_{24}$ The above assumptions are reasonable as observed in Ref. [1]. At $\gamma_{0}$, the initial minimum ${ }_{25}$ coordinate is $q_{0}^{*}$, and we set $q_{0}^{*}=0$ to simplify the notation. Then, $\Delta q^{*}=q^{*}-q_{0}^{*}=q^{*}$. Two ${ }_{26}$ stationary points, the initial minimum and saddle point at strain $\Delta \gamma$, can be obtained by 27 solving the following equation

$$
\frac{\partial U}{\partial q^{*}}=\frac{\eta^{*}}{2} q^{* 2}+\lambda^{*} q^{*}+V \Delta \gamma \frac{\partial \tau\left(\theta_{\mathrm{L}}\right)}{\partial q^{*}}=0 .
$$

${ }_{28}$ At triggering strain $\Delta \gamma_{c}$, the two roots of Eq. (2) are equal, and

$$
\Delta \gamma_{c}\left(\theta_{\mathrm{L}}\right)=\frac{\lambda^{* 2}}{2 \eta^{*} V \frac{\partial \tau\left(\theta_{\mathrm{L}}\right)}{\partial q^{*}}}
$$

${ }_{29} \Delta \gamma_{c}\left(\theta_{\mathrm{L}}\right)$ reaches minimum when $\frac{\partial \tau\left(\theta_{\mathrm{L}}\right)}{\partial q^{*}}$ reaches maximum. This occurs when the correspond30 ing orientation $\theta_{\mathrm{L}}$ equals to $\theta_{s}$, which is the softest shear orientation defined in the main 31 text. From stress transformation rules, we can get

$$
\frac{\partial \tau}{\partial q^{*}}\left(\theta_{\mathrm{L}}\right)=\frac{\partial \tau\left(\theta_{s}\right)}{\partial q^{*}} \cdot \cos \left[2\left(\theta_{\mathrm{L}}-\theta_{s}\right)\right] .
$$

${ }_{32}$ Finally, the relation between $\Delta \gamma_{c}$ and the angle can be written as

$$
\Delta \gamma_{c}=\Delta \gamma_{c, \min } \cdot \cos \left[2\left(\theta_{\mathrm{L}}-\theta_{s}\right)\right]
$$




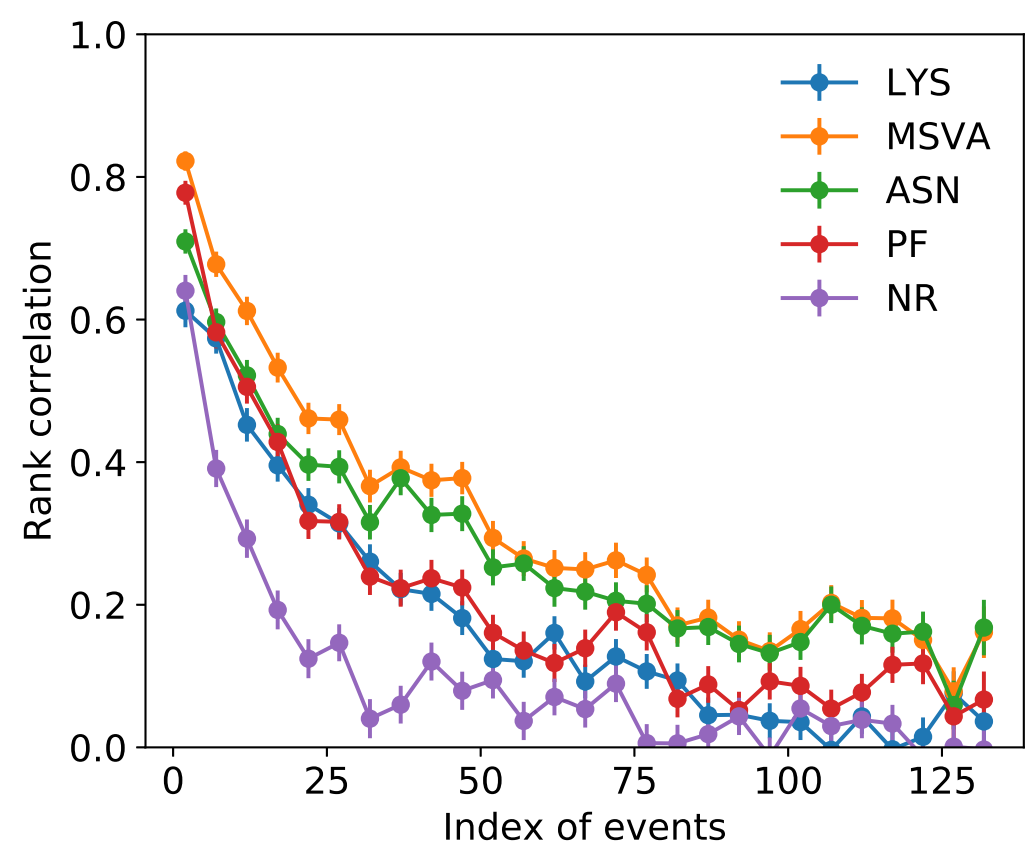

FIG. S2. Correlation between different indicators and the locations of plastic events as a function of index of events.

\section{зз III. PREDICTIVE POWER OF INDICATORS FOR CONFIGURATIONS IN- ${ }_{34}$ STANTLY QUENCHED FROM HIGH TEMPERATURE LIQUIDS}

35 In the main text, we show the predictive power of indicators for configurations prepared ${ }_{36}$ via a slow cooling rate from high temperature liquid. Here, we show the results for config37 urations prepared via instantly quenching from high temperature liquids (HTL) in Fig. S2. ${ }_{38}$ Different from the those slowly quenched configurations, MSVA and ASN have a better 39 predictive power than the LYS. One possible reason for the big drop of predictive power of 40 LYS is that the LYS is purely a local method. Its statistics is narrower for HTL and has the ${ }_{41}$ same order as the mechanical noise [2].

\section{IV. LOCATIONS OF PLASTIC EVENTS WITH DIFFERENT SHEAR PROTO- ${ }_{43}$ COLS}

$44 \quad$ In the Fig. 2 of the main text, we show the correlation between $\theta_{s}$ calculated from the 45 undeformed configuration and the locations of plastic events in shear protocols with $\theta_{\mathrm{L}}=0$ 
(a)

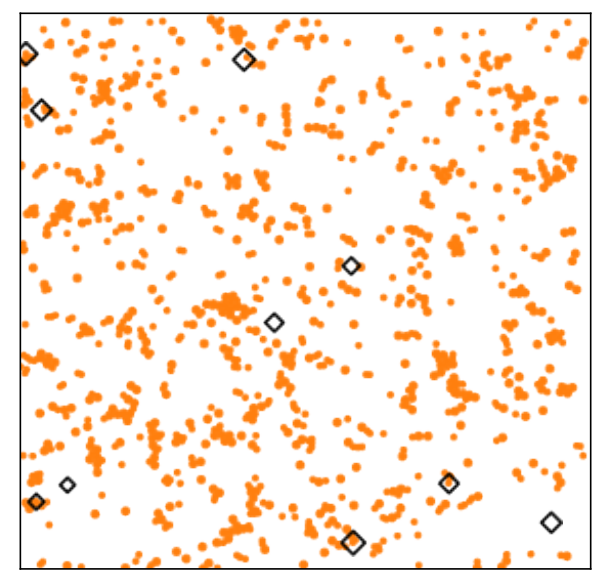

(b)

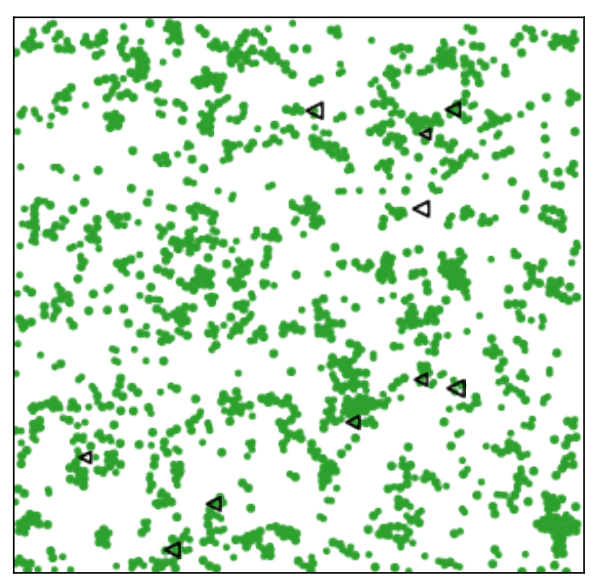

FIG. S3. (a) Orange circles represents the atom with $\mathrm{RC}_{\hat{G}}>0$ and $\theta_{s}>0$. Diamond mark the locations of the first ten plastic events with $\theta_{\mathrm{L}}=\frac{\pi}{4}$. (b) Green circles represents the atom with $\mathrm{RC}_{\hat{G}}>0$ and $\theta_{s}<0$. Triangles mark the locations of the first ten plastic with $\theta_{\mathrm{L}}=-\frac{\pi}{4}$.

46 and $\frac{\pi}{2}$. Here in Fig. S3, we show the results of the same configuration for two other shear 47 protocols with $\theta_{\mathrm{L}}=\frac{\pi}{4}$ and $-\frac{\pi}{4}$. It was observed that core atoms of most events satisfied the ${ }_{48}$ condition $\left|\theta_{s}-\theta_{\mathrm{L}}\right|<\frac{\pi}{4}$ mentioned in the main text.

\section{${ }_{49} \mathrm{~V}$. STATISTICS FOR THE ROTATION OF THE SOFTEST SHEAR ORIENTA- ${ }_{50}$ TION}

51 In the main text, we show that the distribution of the softest orientations is broadened 52 when measured from the configuration associated with the previous event. Here, we show ${ }_{53}$ that the broadening effect is correlated with incremental triggering strain required to induce ${ }_{54}$ each event. As shown in Fig. S4(a), the fraction of the plastic events with orientations within 55 the range $\theta_{s}\left(\mathrm{ID}_{N}, \gamma_{c, N-1, A}\right)-\theta_{\mathrm{L}}<\frac{\pi}{4}$ decreases from one to around 0.75. We also measure ${ }_{56}$ the distribution of the change of orientation for the core atoms for each plastic event since ${ }_{57}$ the triggering of the previous event, as shown in Fig. S4(b). The change of orientation for 58 most events are small, but a few events do undergo large change of orientation. 

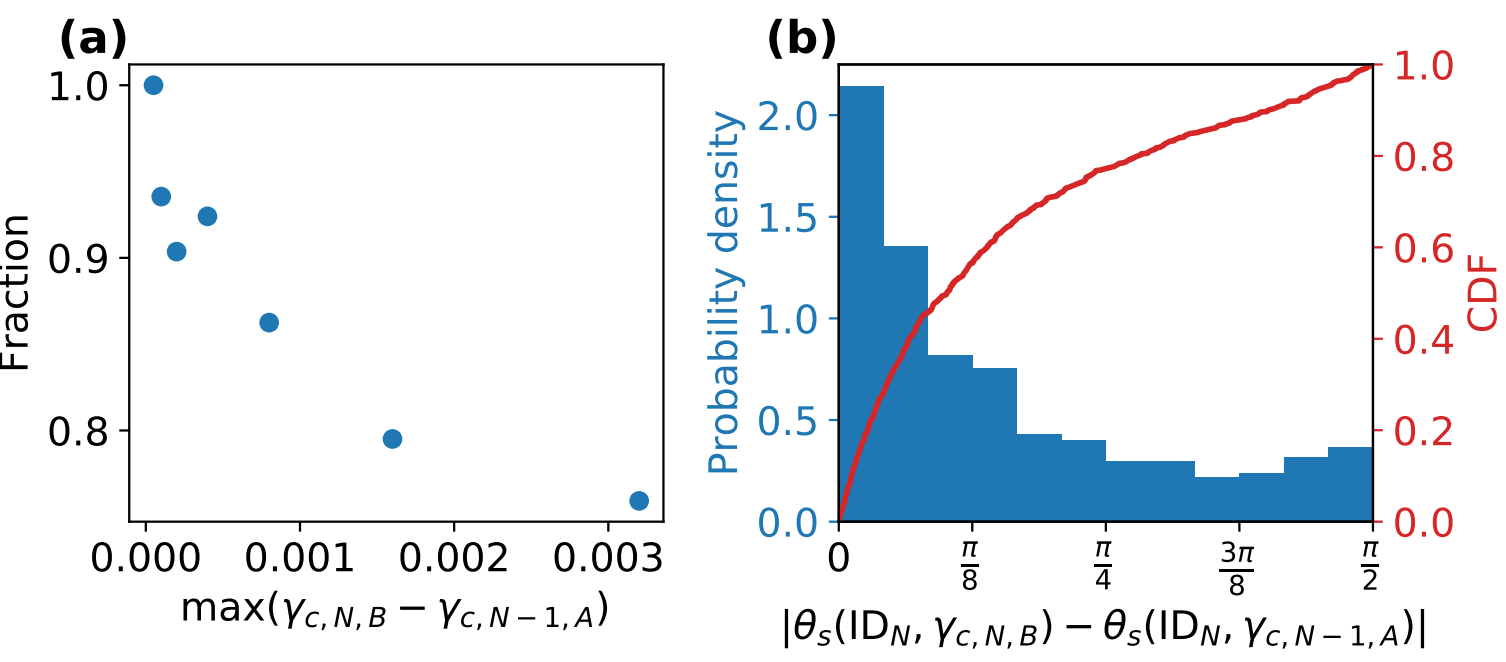

FIG. S4. (a) Measuring the broadening effect of distribution of orientation of plastic events at different strains intervals before triggering point. The fraction is the number fraction of events that satisfy $\left|\theta_{s}\left(\mathrm{ID}_{N}, \gamma_{c, N-1, A}\right)-\theta_{\mathrm{L}}\right|<\frac{\pi}{4}$. If one measure the orientations of the plastic events at a larger strain before the triggering point, the distribution of orientations will be more broaden. (b) The change of orientations if one measure the orientation of plastic events from the configurations after the triggering of previous event of each event and from the configuration just before the triggering of each event. Red line represents the commutative distribution function of the distribution.

\section{${ }_{59}$ VI. IMPROVING THE PRECISION OF PREDICTION VIA CHARACTERIZING 60 THE SOFTEST SHEAR ORIENTATION}

${ }_{61}$ As the plastic events tend to happen in STZs with the softest shear orientations close ${ }_{62}$ the orientation of shear protocols, one can expect that characterizing the softest shear ori${ }_{63}$ entations will help improve the predictive power of those indicators that do not contain ${ }_{64}$ orientational information. In Fig. S5, we show that the precision of predicting the location 65 of core atoms for first events of 100 samples is improved by a factor close to 2 by filtering ${ }_{66}$ out those regions that do not have a good alignment with the orientations with the shear ${ }_{67}$ protocols. In the graph, the rank threshold means that the threshold to label the "softest" ${ }_{68}$ atoms characterized by different indicators as the potential locations of first plastic event of ${ }_{69}$ each sample. E.g. if rank threshold equals 10 for atomic shear shear nonaffinity, we label 70 the atoms with the 10 lowest atomic shear nonaffinity as the potential locations of the first 


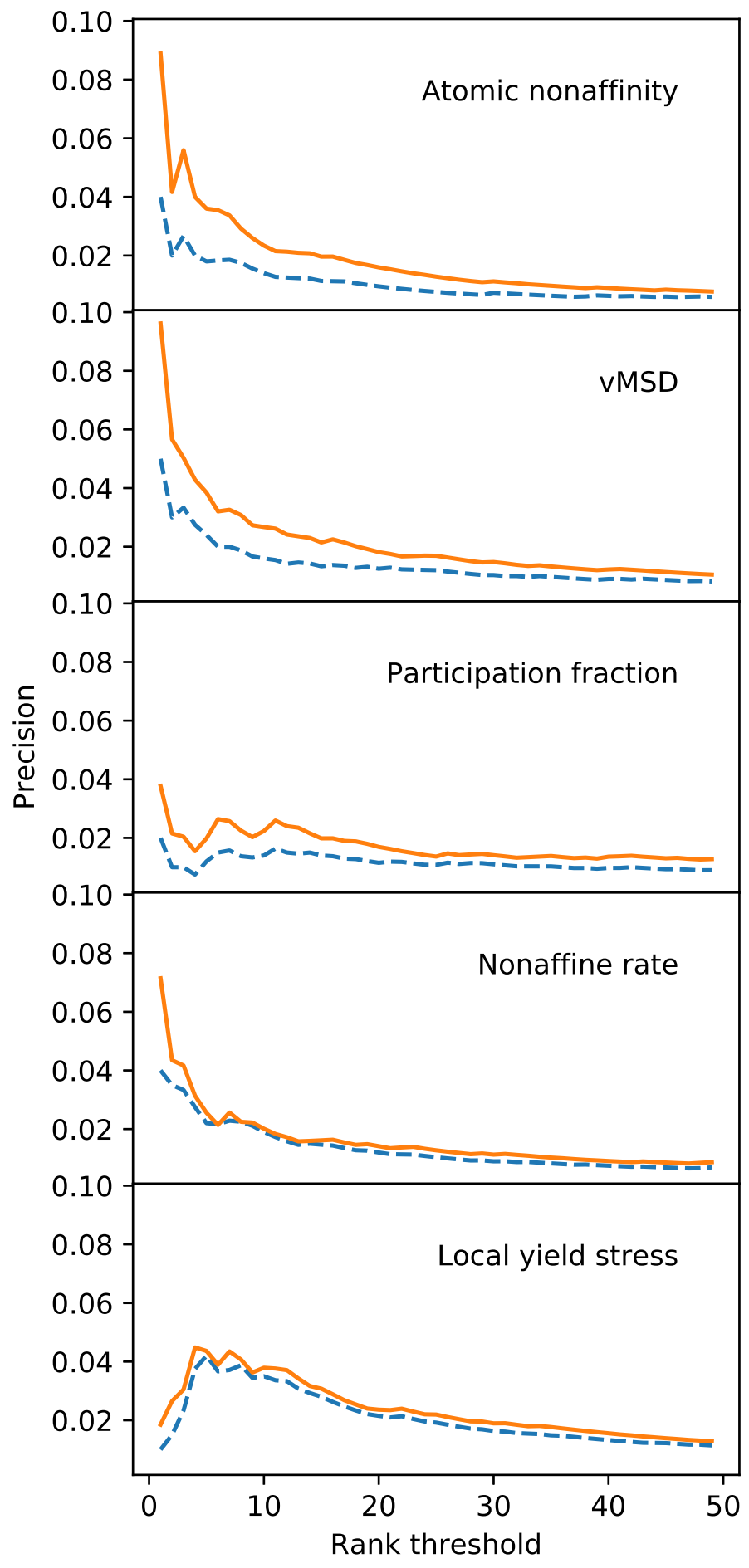

FIG. S5. Predictive precision of different indicators at different rank threshold. Dash blue lines represent results for single indicators. Solid orange lines represent results for each indicators after filtering the atoms with $\left|\theta_{S}-\theta_{\mathrm{L}}\right|<\pi / 4$. 
71 plastic events. For 100 samples we used here, we will totally label 1000 atoms. If in all 72 labeled atoms, there are 30 atoms that are just the core atoms of the 100 first plastic event 73 of 100 samples, the precision will be $30 / 1000=0.03$, which is True Positive/ Total postive. 74 After obtaining the softest shear orientational of each atom, for those 1000 labeled atoms, 75 we filter those atoms with $\left|\theta_{S}-\theta_{\mathrm{L}}\right|<\pi / 4$, since we estimate that only the plastic events will 76 in most cases happen in those regions that have a good alignment with shear protocols. On 77 average, 500 atoms will left after filtering, since the amorphous materials are statistically 78 isotropic in the quench state. If all the true positive atoms are in the atoms left after filter$79 \mathrm{ing}$, the precision will be $30 / 500=0.06$, where the precision will be improved by a factor 80 of 2 . In Fig. S5, the improving factor is close to 2 for small rank threshold but decay to 81 smaller values as rank threshold increases.

\section{VII. A SIMPLE MODEL OF INDEPENDENT PLASTIC EVENTS WITH IN- 83 TRINSIC ORIENTATIONS}

84 In this section, we present a simple mean-field model of independent plastic events with 85 intrinsic orientations to understand the distribution of orientations for triggered plastic ${ }_{86}$ events after shear deformation.

\section{A. Assumptions}

- Each shear transformation zone (STZ) in an amorphous solid has two important and independent properties, i.e. the softest shear orientation (denoted as $\theta_{s, i}$ ) and the minimum triggering strain (denoted as $\left.\gamma_{c, s, i}\right) . \quad \theta_{s, i}$ is confined in the range $\left[-\frac{\pi}{2}, \frac{\pi}{2}\right.$ ) considering the symmetry of shear.

- The triggering strain for one STZ at a given imposed shear orientation (denoted as $\left.\theta_{\mathrm{L}}\right)$ may be expressed as

$$
\gamma_{c, i}\left(\theta_{\mathrm{L}}\right)=\frac{\gamma_{c, s, i}}{\cos \left[2\left(\theta_{s, i}-\theta_{\mathrm{L}}\right)\right]}
$$

- The initial microstructure of the amorphous solid is isotropic. Then, the number density of STZs with specified properties $\theta_{s}$ and $\gamma_{c, s}$ does not rely on the $\theta_{s}$, i.e.

$$
\rho\left(\theta_{s}, \gamma_{c, s}\right)=\rho\left(\gamma_{c, s}\right)
$$



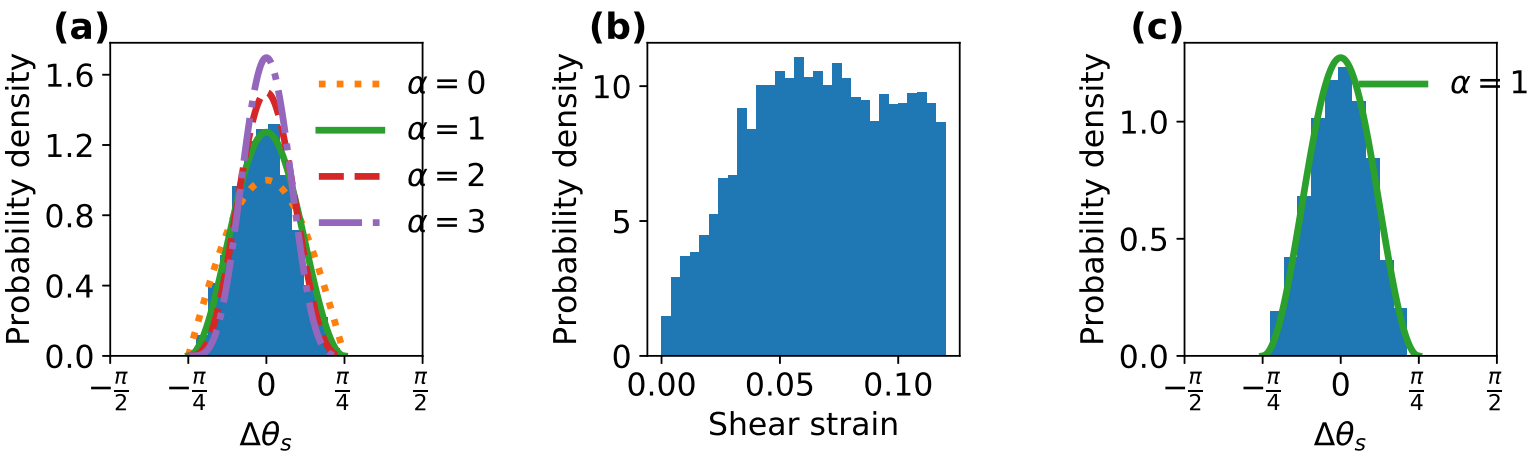

FIG. S6. (a) The distribution of the softest shear orientations for all the plastic events that are triggered before shear strain 0.12 in 10 samples. The softest shear orientations are measured based on the configuration just before the triggering of each event. Each line represents the predictions from Eqs. (11) and (12). (b) The probability density for the triggering strains for all the plastic events in AQS for 100 samples. (c) The distribution of the softest shear orientations for the first five events that are triggered in AQS for 100 samples. The softest shear orientations are measured based on the configuration just before the triggering of each event.

96 where $\rho$ is the number density.

- We further assume that $\rho\left(\gamma_{c, s}\right)$ follows a power law, i.e. $\rho\left(\gamma_{c, s}\right)=A \gamma_{c, s}^{\alpha}$.

98 Noting that here we neglects that fact that when one STZ is activated upon deformation 99 it may get a new orientation and new minimum triggering strain. This is reasonable when 100 new triggering strain is large and plastic events are sparse, which is close to the situation 101 of that the configurations are prepared with slow cooling rate and observing strain range is 102 before yielding. ${ }_{104}$ orientations of triggered events in one shear orientation

We set the orientation of shear protocol as the referential orientation with value of $\theta_{\mathrm{L}}=0$, 106 set the loading shear strain as $\gamma_{\mathrm{L}}$ and denote the total number of the plastic events with 
${ }_{107} \gamma_{c, s} \leq \gamma_{\mathrm{L}}$ as $N\left(\gamma_{\mathrm{L}}\right)$. Then, we obtain

$$
N\left(\gamma_{\mathrm{L}}\right)=\int_{\frac{\pi}{2}}^{-\frac{\pi}{2}} \int_{0}^{\gamma_{\mathrm{L}}} \rho\left(\gamma_{c, s}\right) d \gamma_{c, s} d \theta_{s}=\pi \int_{0}^{\gamma_{\mathrm{L}}} \rho\left(\gamma_{c, s}\right) d \gamma_{c, s}=\frac{A \pi}{\alpha+1} \gamma_{\mathrm{L}}^{\alpha+1}
$$

108 Then,

$$
A=\frac{(\alpha+1) N\left(\gamma_{\mathrm{L}}\right)}{\pi \gamma_{\mathrm{L}}^{\alpha+1}}
$$

${ }_{109}$ For events with $\left|\theta_{s}\right| \leq \frac{\pi}{4}$, only those events with condition that $\gamma_{c, s} \leq \gamma_{\mathrm{L}} \cos 2 \theta_{s}$ will triggered. ${ }_{110}$ Then, the number density $(\tilde{\rho})$ of events that are triggered with property $\theta_{s}$ is

$$
\tilde{\rho}\left(\theta_{s}\right)=\int_{0}^{\gamma_{\mathrm{L}} \cos 2 \theta_{s}} \rho\left(\gamma_{c, s}\right) d \gamma_{c, s}=\frac{A}{\alpha+1} \gamma_{\mathrm{L}}^{\alpha+1} \cos ^{\alpha+1} 2 \theta_{s}=\frac{N\left(\gamma_{\mathrm{L}}\right)}{\pi} \cos ^{\alpha+1} 2 \theta_{s}
$$

${ }_{111}$ Events with $\left|\theta_{s}\right|>\frac{\pi}{4}$ will not be triggered in this protocol. Thus, the probability density ${ }_{112}\left(\hat{\rho}\left(\theta_{s}\right)\right)$ of events that are triggered should have the form

$$
\hat{\rho}\left(\theta_{s}\right)=k \cos ^{\alpha+1} 2 \theta_{s},
$$

113 where $k$ is the normalizing factor that ensures that the total probability equals one. The ${ }_{114}$ peak probability corresponds to events that have the same orientation as the shear protocol 115 i.e. $\theta_{s}=0$, and $\hat{\rho}_{\max }=\hat{\rho}\left(\theta_{s}=0\right)=k$. For different power-law factors, we infer the value of ${ }_{116} k$ to be

$$
\begin{aligned}
& \alpha=0, k=1, \\
& \alpha=1, k=\frac{4}{\pi} \approx 1.27, \\
& \alpha=2, k=\frac{3}{2}, \\
& \alpha=3, k=\frac{16}{3 \pi} \approx 1.70 .
\end{aligned}
$$

117 In the systems that we investigated in the main text, we found that $\hat{\rho}\left(\theta_{s}\right)$ corresponds closely 118 to what would be expected from $\alpha=1$, as shown in Fig. S6(a). When $\alpha=1$, one can also 119 derive that the probability density of triggering strains for the plastic events in AQS should 120 be a linear function of shear strain; this was further verified in simulations as shown in ${ }_{121}$ Fig. S6(b). The function is close to linear relation before yielding, but a plateau arises after 122 yielding. To confirm the linear regime we further checked the distribution of the softest 123 orientations for the first five plastic in 100 samples. The distribution was also found to be 
${ }_{124}$ close to distribution of $\alpha=1$, as shown in Fig. S6(c).

125 [1] B. Xu, M. Falk, J. Li, and L. Kong, Phyiscal Review B 95, 144201 (2017).

126 [2] A. Barbot, M. Lerbinger, A. Hernandez-Garcia, R. García-García, M. L. Falk, D. Vandem-

127 broucq, and S. Patinet, Physical Review E 97, 033001 (2018), arXiv:1802.05796. 